Archives

$8 \mid 1991$

Varia

\title{
Le groupe de recherches sur les images médiévales
}

Jean-Claude Schmitt

\section{(2) OpenEdition}

Journals

Édition électronique

URL : http://journals.openedition.org/ccrh/2820

DOI : $10.4000 /$ ccrh. 2820

ISSN : $1760-7906$

Éditeur

Centre de recherches historiques - EHESS

Édition imprimée

Date de publication : 15 octobre 1991

ISSN : 0990-9141

Référence électronique

Jean-Claude Schmitt, "Le groupe de recherches sur les images médiévales », Les Cahiers du Centre de Recherches Historiques [En ligne], 8 | 1991, mis en ligne le 18 mars 2009, consulté le 10 décembre 2020. URL : http://journals.openedition.org/ccrh/2820 ; DOI : https://doi.org/10.4000/ccrh.2820

Ce document a été généré automatiquement le 10 décembre 2020.

Article L.111-1 du Code de la propriété intellectuelle. 


\title{
Le groupe de recherches sur les images médiévales
}

\author{
Jean-Claude Schmitt
}

1 Au sein du Groupe d'Anthropologie Historique de l'Occident Médiéval (qui poursuit par ailleurs sa vaste enquête sur la littérature médiévale des exempla ${ }^{1}$ ), a été créé il y a six ans ce que nous nommons entre nous le «Groupe Images ». Y travaillent en permanence, outre une poignée d'enseignants et de chercheurs ${ }^{2}$, une vingtaine d'étudiants en thèse. Le but de ce groupe est triple :

- documentaire : il s'agit de fournir aux chercheurs une documentation iconographique riche, variée et facilement accessible ;

- scientifique : en associant étroitement la constitution de cette documentation aux recherches collectives ${ }^{3}$ ou individuelles des chercheurs et des étudiants ;

- pédagogique : non seulement en se donnant un matériel documentaire utilisable dans les séminaires, mais en associant étroitement les étudiants à la constitution d'une iconothèque. Notre souci constant est de faire progresser du même pas la recherche et la formation à la recherche.

2 Les activités du Groupe comportent actuellement deux volets :

- la poursuite de la constitution et de l'indexation sur ordinateur d'une iconothèque ;

- la réalisation d'un vidéodisque en collaboration avec l'École Française de Rome et la Bibliothèque Vaticane.

\section{Une iconothèque informatisée}

3 La constitution de l'iconothèque a commencé dès l'origine du Groupe. Elle concerne presque exclusivement les miniatures médiévales et consiste en diapositives (environ 6000 aujourd'hui). Dans la mesure du possible, nous cherchons à obtenir des reproductions de manuscrits complets, avec toutes les miniatures qu'ils contiennent. Dans le choix des manuscrits, nous visons à la diversité des oeuvres, des époques et des provenances géographiques. Le choix des manuscrits dépend aussi pour beaucoup des recherches menées par les uns et les autres. Notre fonds est particulièrement riche pour 
les manuscrits de la Bodleian Library d'Oxford, de la Staatsbibliothek de Munich (entre autres les grands manuscrits ottoniens) et des bibliothèques parisiennes. Près de 300 manuscrits ont été enregistrés à l'heure actuelle.

Volontairement, nous avons commencé l'indexation de manière empirique et tâtonnante, pour parvenir progressivement à un thesaurus à peu près clos d'environ 1300 termes $^{4}$. Il nous a semblé indispensable de constituer notre propre thesaurus descriptif, faute d'en trouver un tout fait, qui fût à la fois adapté à notre matériel documentaire et assez simple pour permettre une indexation rapide de nos miniatures. C'est pourquoi, tout en en tirant d'utiles enseignements, nous nous sommes détournés à la fois du Thesaurus de François Garnier, dont les classifications hiérarchisées se prêtaient mal à nos principes d'indexation, et du système néerlandais Iconclass, trop lourd et d'un emploi encore plus malaisé.

5 A chacune de nos diapositives correspond, sous le même numéro, une fiche informatisée qui ne retient que les caractères externes de la figuration, sans en proposer d'interprétation. Le but est en effet de permettre de retrouver aisément des images, non de se substituer au chercheur et encore moins de lui imposer nos jugements subjectifs. La fiche d'indexation propose ainsi une vingtaine de rubriques :

- le numéro de la diapositive ;

- les données relatives à l'identité du manuscrit : la bibliothèque où il est conservé, la cote $\mathrm{du}$ manuscrit, le nom éventuel du fonds, la date (par quart de siècle), l'origine géographique (par pays), le titre de l'oeuvre (Légende dorée, Cité de Dieu, Apocalypse, Roman de la Rose, etc.) ;

- les traits de la description. Chaque rubrique peut accueillir jusqu'à une douzaine de mots au moins, permettant une interrogation sur chacun d'eux ou sur plusieurs d'entre eux simultanément (interrogation de type et/ou). Ce sont: la référence textuelle de l'image (particulièrement précieuse dans le cas de l'iconographie biblique); les «thèmes » de l'image, traduits sous forme de noms propres ou de noms communs (ex. : Annonciation, Adoration des Mages, combat, serment, etc.); les personnages: personnages divins (Dieu-Père, Christ, Trinité), personnages surnaturels (Satan, démons, anges), saints (Vierge, Jean-Baptiste, Jean-Evangéliste), autres personnages (ex: roi, charpentier, Charlemagne). D'autres rubriques concernent les lieux nommés (ex : Rome, Jérusalem, paradis, enfer), les objets (ex : épée, lit, livre), les éléments de la nature (ex : cheval, montagne, lune);

- enfin, deux rubriques sur lesquelles, en principe, l'interrogation ne porte pas, contiennent l'une des remarques complémentaires sur l'image et les inscriptions qu'elle peut contenir, l'autre une légende discursive synthétique, bien utile lorsque le sens général de l'image ne peut être déduit immédiatement de la juxtaposition des mots de la description.

Petit à petit, cette iconothèque et sa base de données sont devenues des instruments de travail utilisés avec d'autant plus de profit par les membres du Groupe qu'ils les avaient eux-mêmes réalisés. A l'intérêt scientifique de l'entreprise sont venus s'ajouter d'autres avantages encore :

- permettre la préparation à l'École, depuis quelques années, de toute une série de thèses de doctorat en histoire médiévale utilisant largement le document iconographique ${ }^{5}$;

- remplir une fonction de sociabilité : en dépit des séminaires, nos étudiants risquent souvent de se sentir isolés dans leur travail, d'autant plus que beaucoup viennent de province ou de l'étranger. Le « Groupe Images » leur permet de se retrouver, de discuter 
librement entre eux et avec les enseignants, de commenter des images autour d'une tasse de café...

- assurer une formation concrète à la recherche iconographique, dont la qualité a été reconnue par plusieurs institutions qui se sont attiré le concours de certains de nos étudiants. Par exemple, leurs thèses soutenues, Brigitte Buettner a pu participer pendant deux ans à la réalisation d'une iconothèque à la Fondation Paul Getty et Odile Blanc travaille actuellement à Rome dans l'équipe du vidéodisque de la Bibliothèque Vaticane. Plusieurs anciens étudiants ont trouvé des postes d'enseignants et de chercheurs en France comme à l'étranger ${ }^{6}$. Enfin, les principes d'indexation que nous avons définis ont inspiré le travail similaire qu'une équipe, comptant plusieurs de nos étudiants, réalise sur les bois gravés des livres anciens de la Bibliothèque Nationale ${ }^{7}$. Ces principes ont surtout été repris presque sans changement pour la réalisation du Vidéodisque de la Bibliothèque Vaticane.

\section{Le vidéodisque de la bibliothèque vaticane}

7 Cette deuxième entreprise, étroitement liée à la première pour la réalisation de son thesaurus, est placée sous la direction de Jérôme Baschet. Quatre de nos étudiants, rémunérés avec des vacations, y travaillent à tour de rôle, en étroite relation avec l'équipe de deux personnes chargée, à Rome, du repérage des manuscrits à peinture et de la préparation des campagnes photographiques ${ }^{8}$. Il s'agit dans l'immédiat de fabriquer et de commercialiser trois vidéodisques, dont le premier verra le jour à la fin de l'année 1991. Il mettra à la disposition du public (et de nous-mêmes), toutes les miniatures des manuscrits de fonds de la Reine Christine (plus de 2000 manuscrits, 20000 clichés environ). Suivront le fonds Urbinate et une partie du fonds Vatican latin. Pour chaque miniature, ce sont au moins deux sinon trois vues différentes qui peuvent apparaitre à l'écran : la pleine page, la miniature complète, éventuellement un détail remarquable. Il n'est pas besoin de s'étendre longuement sur la révolution que représente cette technique dans la recherche documentaire: sur le plan quantitatif (en permettant à n'importe qui la consultation presque instantanée de milliers d'images), et sur le plan qualitatif, puisque le vidéodisque nous fait découvrir des fonds d'images immenses et jusqu'alors très largement inexplorés; quant au chercheur, il aura la possibilité de faire défiler à l'écran et de regrouper selon les critères de son choix des masses sans précédent d'images en couleur provenant de manuscrits différents: on devine les possibilités nouvelles offertes à la constitution de séries d'images et à la comparaison des images entre elles, bases du travail iconographique. Tout cela est inconcevable dans le cadre d'une consultation in situ des manuscrits et même dans celui d'une iconothèque traditionnelle.

8 Sans dissimuler les difficultés (notamment financières ${ }^{9}$ ) de l'activité aux multiples facettes du «Groupe Images", nous voudrions pour finir souligner la satisfaction que donne l'exploration de techniques nouvelles considérées non en elles-mêmes, mais en tant qu'outils immédiatement mis à profit dans la recherche scientifique et en tant qu'instruments de formation des étudiants. 


\section{NOTES}

1. Cette autre enquête est principalement animée par Claude Brémond, Jacques Berlioz (CNRS), Marie-Anne Polo de Beaulieu (CNRS), Colette Ribaucourt. La publication par des membres de cette équipe de plusieurs recueils d'exempla (celui de Jean Gobi, l'Alphabetum Narrationum, une partie de celui d'Etienne de Bourbon) est imminente.

2. Placés sous la direction de Jean-Claude Schmitt, Jean-Claude Bonne, Jérôme Baschet ainsi que Michel Pastoureau (IVe Section de l'EPHE), assistés depuis 1990-91 d'Aline Debert. Anton Schütz, chargé de conférences, lui prête également son concours.

3. A ses débuts, le Groupe a participé aux deux Actions Thématiques Programmées du CNRS : «Polythéismes » et «Genèse de l'État moderne ». Ultérieurement, les activités du Groupe ont bénéficié d'une aide financière du CENCEP.

4. En 1985, la base informatique a été établie sur un ordinateur Apple II qui s'est révélé de plus en plus insuffisant. Le transfert sur PC avec un programme de base de données Texto et un disque dur de 40 Mo, a été accompli en 1990. Nous avons particulièrement bénéficié pour cela de l'aide de MarieChristine Vouloir (Centre de Démographie Historique) et de Jean-Paul Coulier. Nous tenons aussi à exprimer notre gratitude, pour son constant soutien, à Anne-Marie Meunier.

5. Les thèses préparées dans ce cadre et soutenues ces dernières années sont celles de Jérôme Baschet (Les Justices de l'au-delà), Christine Lapostolle (Les représentations du désert au Moyen Age), Brigitte Buettner (Les Manuscrits français des «Claires Femmes» de Boccace), Odile Blanc (Corps et vêtement de cours à la fin du Moyen Age en Flandre et en Bourgogne), Colette Deremote (Le P rogramme des grandes verrières de Chartres), Philippe Buc (Exégèse et idéologie politique, XII ${ }^{\mathrm{e}}$-XIII siècles). Les thèses en cours d'achèvement sont celles de: Marie-Laure Le Bail (L'Iconographie juridique dans les coutumiers du Midi), Anca Bratu (Les Lieux intermédiaires de l'au-delà), Philippe Faure (Les anges), Pierre Bureau (Le thème du dépouillement dans les images médiévales), Isabelle Toinet (Le Symbolisme de la bouche), Isabelle Marchesin (Sons et voix dans les images médiévales), Cécile Quentel (La Souffrance des femmes), Véronique Frandon (Les images des saisons), Sylvie Chossat (L'Iconographie arthurienne).

6. Outre l'élection récente de Jérôme Baschet à l'EHESS, signalons, en Histoire de l'Art, celles de Colette Deremble à l'Université de Paris X-Nanterre et de Brigitte Buettner à Smith College (Northampton, Massachussets), et en Histoire médiévale, celle de Philippe Buc à l'Université Standford (Californie).

7. La conception de ce projet, sous la direction de M. Daniel Toulet, Conservateur de la Réserve des Imprimés de la BN, doit beaucoup à Danièle Sansy.

8. Il s'agit de Christiane Baryla, Conservateur détaché de la Bibliothèque Sainte-Geneviève, et d'Odile Blanc, membre de longue date du «Groupe Images ». La réalisation du vidéodisque n'est possible que grâce au mécénat du Groupe UAP.

9. Cela essentiellement en raison du renchérissement du prix des diapositives acquises dans les bibliothèques publiques, notamment à la Bibliothèque Nationale. Il est regrettable que celle-ci soit revenue récemment à une pratique que l'on croyait abolie, qui consiste à surfacturer les nouveaux clichés par rapport à la reproduction des clichés existants. On pénalise ainsi les chercheurs tout en encourageant la tendance fâcheuse des agences photographiques à toujours republier les mêmes images. 


\section{AUTEUR}

JEAN-CLAUDE SCHMITT

Jean-Claude SCHMITT est directeur d'études à l'EHESS et membre du Centre de Recherches Historiques. 